\title{
Fungos micorrízicos e fósforo, no crescimento, nos teores de nutrientes e na produção do sorgo e soja consorciados(1)
}

\author{
Wellington Bressan ${ }^{(2)}$, José Oswaldo Siqueira( ${ }^{(3)}$, Carlos Alberto Vasconcellos ${ }^{(2)}$ e Antonio Alvaro Corsetti Purcino(2)
}

\begin{abstract}
Resumo - O objetivo deste trabalho foi avaliar em casa de vegetação os efeitos da inoculação de fungos micorrízicos arbusculares sobre o crescimento, produção de grãos e teores de nutrientes no sorgo, e na soja, consorciados, num Latossolo Vermelho-Escuro fumigado que recebeu 0, 25, 50, 100 e $200 \mathrm{mg} / \mathrm{kg}$ de P. O peso da matéria seca da parte aérea do sorgo aumentou em 55\%, 33\% e 6\%, respectivamente, com o Glomus etunicatum, o Gigaspora margarita e o Glomus clarum. O peso dos grãos secos aumentou em $31 \%$ e 21\%, respectivamente, com o Glomus etunicatum e com o Gigaspora margarita. Na soja, o peso da matéria seca da parte aérea aumentou linearmente com as doses de $\mathrm{P}$, independentemente da inoculação de fungo micorrízico, enquanto o peso dos grãos secos aumentou na mesma grandeza que o do sorgo. O Glomus etunicatum foi a espécie micorrízica mais eficiente em todas as doses de P. A inoculação de fungos micorrízicos contribuiu para o aumento da matéria seca, produção de grãos e para os teores dos nutrientes $\mathrm{N}, \mathrm{P}, \mathrm{K}, \mathrm{Zn}$ e Cu no sorgo e na soja. Estes efeitos são dependentes das doses de $\mathrm{P}$ no solo e da espécie de fungos micorrízicos inoculados.
\end{abstract}

Termos para indexação: micorriza, consorciação, matéria seca, absorção de nutrientes.

\section{Mycorhizal fungi and phosphorus on growth, yield and nutrition of intercropped grain sorghum and soybean}

\begin{abstract}
The objective of this research was to evaluate the effect of three endomycorhizal fungi species on intercropped sorghum and soybean growth, nutrition and grain yield in a Dark-Red Latosol (Typic haplusthox) fumigated soil amended with $0,25,50,100$ and $200 \mathrm{mg} / \mathrm{kg}$ of P. The experiment was conducted under greenhouse conditions. Sorghum shoot dry weight increased by 55\%,33\% and $6 \%$, and grain dry weight increased by $31 \%$ and $21 \%$, respectively to Glomus etunicatum and Gigaspora margarita. Glomus clarum did not statistically differ from the control. Soybean shoot dry weight and grain dry weight showed linearly increase with P levels indepedently of mycorhizal fungus inoculation, while dry grain weigth incresed in the same way as that of sorghum. Glomus etunicatum was the most efficient mycorhizal fungus among the species at all $\mathrm{P}$ levels. The results demonstrate that mycorhizal inoculation contributed substantially to increase shoot and grain dry weight as well as to increase $\mathrm{N}, \mathrm{P}, \mathrm{K}, \mathrm{Zn}$ and $\mathrm{Cu}$ shoot contents. These responses varied with soil $\mathrm{P}$ levels and mycorhizal species.
\end{abstract}

Index terms: mycorrhizae, intercropping, dry matter, nutrient uptake.

\section{Introdução}

Os fungos que formam as micorrizas arbusculares (MAs) são de ocorrência generalizada na maioria das

(1) Aceito para publicação em 4 de outubro de 2000.

Extraído da dissertação de doutorado, apresentada pelo primeiro autor à Universidade Federal de Lavras (UFLA), Lavras, MG.

${ }^{(2)}$ Embrapa-Centro Nacional de Pesquisa de Milho e Sorgo (CNPMS), Caixa Postal 151, CEP 35701-970, Sete Lagoas, MG. E-mail:bressan@cnpms.embrapa.br, carlos@cnpms.embrapa.br, corsetti@cnpms.embrapa.br

(3)UFLA, Dep. de Ciência do Solo, Caixa Postal 37, CEP 37200-000, Lavras, MG. espécies de plantas, e não apresentam especificidade hospedeira; mas sua capacidade em promover o crescimento da planta pode variar em razão do fungo, da planta, e do ambiente (Smith \& Gianinazzi-Pearson, 1988). Isto ocorre devido a diferenças no grau de infectividade, e na eficiência das diferentes espécies de fungos em promover a absorção de $P$ pelas raízes. Esse fato está amplamente documentado em relação a uma imensa variedade de espécies vegetais. Trabalhando em solos de cerrado, Paula et al. (1990) verificaram que diferentes populações de fungos micorrízicos mostraram diferenças na taxa de colonização, de absorção de $\mathrm{P}$, de produção de matéria 
seca da parte aérea, e de produção de grãos da soja. Miranda et al. (1989) mostraram que a inoculação de fungos micorrízicos no sorgo aumentou a produção de matéria seca da parte aérea e os teores de $\mathrm{P}$ e $\mathrm{N}$. Na soja, além dos benefícios diretos, existem efeitos positivos na interação fungo micorrízico-rizóbio, que resultam da melhor nutrição de $\mathrm{P}$, necessária para a nodulação e a fixação biológica de $\mathrm{N}$ (Paula \& Siqueira, 1987; Vejsadova et al., 1992).

Apesar do grande volume de pesquisas sobre $\mathrm{Pe}$ micorrizas em plantas cultivadas, os efeitos desses fatores sobre o consórcio sorgo-soja ainda são muito restritos, principalmente quanto aos fungos micorrízicos, que interferem na capacidade competitiva das plantas e podem atuar de modo diferenciado sobre as espécies consorciadas.

O objetivo deste trabalho foi avaliar o efeito de três espécies de fungos micorrízicos no crescimento, nos teores de nutrientes, e na produção de grãos do sorgo e soja em consorciação, num solo de cerrado com diferentes doses de $\mathrm{P}$.

\section{Material e Métodos}

$\mathrm{O}$ experimento constou de um fatorial ( $4 \times 5)$, sendo três espécies de fungos micorrízicos arbusculares (Glomus etunicatum, Gigaspora margarita e Glomus clarum) e um tratamento sem inoculação, e cinco doses de $\mathrm{P}(0,25,50$, $100,200 \mathrm{mg} / \mathrm{kg}$ de solo), com cinco repetições por tratamento, em delineamento completamente casualizado. Foi conduzido em casa de vegetação, na Embrapa-Centro Nacional de Pesquisa de Milho e Sorgo, Sete Lagoas, MG, de outubro a dezembro de 1994, em Latossolo VermelhoEscuro distrófico, sob vegetação de cerrado, em vasos com $16 \mathrm{~kg}$ de solo. O solo foi coletado a uma profundidade de $0-20 \mathrm{~cm}$ e apresentou as seguintes características: $\mathrm{pH}$ em água $(1: 2,5)=5,1 ; \mathrm{H}+\mathrm{Al}\left(\mathrm{cmol}_{\mathrm{c}} / \mathrm{dm}^{3}\right)=7,92$; $\mathrm{Al}\left(\mathrm{cmol}_{\mathrm{c}} / \mathrm{dm}^{3}\right)=0,80 ; \mathrm{Ca}\left(\mathrm{cmol}_{\mathrm{c}} / \mathrm{dm}^{3}\right)=1,70 \mathrm{e}$ $\mathrm{Mg}\left(\mathrm{cmol}_{\mathrm{c}} / \mathrm{dm}^{3}\right)=0,33$ extraídos com $\mathrm{KCl} 1 \mathrm{~mol} / \mathrm{L} \mathrm{e}$ determinados por espectrometria de absorção atômica; $\mathrm{K}\left(\mathrm{mg} / \mathrm{dm}^{3}\right)=43$ e P $\left(\mathrm{mg} / \mathrm{dm}^{3}\right)=4$, extraídos por Mehlich $1\left(\mathrm{HCl} 0,05 \mathrm{~mol} / \mathrm{L}+\mathrm{H}_{2} \mathrm{SO}_{4}\right.$ 0,0125 mol/L) determinados por fotometria de chama e colorimetria, respectivamente; $\mathrm{MO}(\mathrm{g} / \mathrm{kg})=43,1$, saturação de $\mathrm{Al}(\%)=27$. O solo foi desinfestado com bromex (brometo de metila $98 \%$ + cloropicrina $2 \%$ ), na dosagem de $100 \mathrm{~cm}^{3} / \mathrm{m}^{3}$ de solo, e recebeu calagem com calcário dolomítico Supermil (PRNT $=95 \%$ ), suficiente para elevar o pH para 6,0. Após a adição de calcário, foi efetuada a adubação com nitrato de amônio, superfosfato simples e cloreto de potássio, respectivamente, como fontes de $\mathrm{N}(63 \mathrm{mg} / \mathrm{kg}), \mathrm{P}(25,50$, 100 e $200 \mathrm{mg} / \mathrm{kg})$ e $\mathrm{K}(300 \mathrm{mg} / \mathrm{kg})$, e com FTE BR 12 $(0,05 \mathrm{~g} / \mathrm{kg})$. Após a adição de $\mathrm{P}$ e antes da semeadura, o solo apresentou 6,0, 9,0,14,0 e 34,0 mg/kg de P extraído pelo Mehlich 1, respectivamente, nos tratamentos que receberam 25, 50, 100 e $200 \mathrm{mg} / \mathrm{kg}$ de $\mathrm{P}$.

A semeadura foi efetuada com cinco sementes de sorgo (Sorgum bicolor L. Moench), cultivar BR 304 (ciclo médio), e cinco sementes de soja (Glycine max L.), cultivar Garimpo (ciclo precoce) em cada vaso; o desbaste foi efetuado aos 15 dias após a emergência, deixando-se uma planta de sorgo e uma de soja por vaso. A inoculação de fungos micorrízicos nas plantas foi efetuada aplicando-se $10 \mathrm{~mL}$ de uma suspensão de esporos contendo 500 esporos por vaso. Os vasos sem inoculação de fungos micorrízicos receberam $30 \mathrm{~mL}$ de um filtrado dos inóculos provenientes dos solos utilizados na multiplicação dos fungos. Os esporos, extraídos por peneiramento, via úmida (Gerdemann \& Nicholson, 1963), foram contados e separados sob microscópio estereoscópio, colocados em papel de filtro, e armazenados a $4^{\circ} \mathrm{C}$ até a sua utilização como inóculo. As sementes de soja receberam inóculo de Bradyrhizobium japonicum, à base de turfa, sendo aplicados $4 \mathrm{~g} / \mathrm{g}$ de semente.

A irrigação dos vasos foi efetuada em razão de necessidade, com base no peso dos vasos, mantendo-se $60 \%$ do volume total de poros preenchidos com água. As condições na casa de vegetação foram: temperatura máxima $34^{\circ} \mathrm{C}$, temperatura mínima $22^{\circ} \mathrm{C}$ e luminosidade média de $625 \mu$ eisteins $/ \mathrm{m}^{2} / \mathrm{seg}$. O N foi aplicado em cobertura 25 dias após o desbaste, na concentração de $2,88 \mathrm{~g}$ de $\mathrm{NH}_{4} \mathrm{NO}_{3} / \mathrm{L} /$ vaso.

$\mathrm{O}$ experimento foi conduzido por 105 dias e colhido quando o sorgo apresentou o estádio de grão pastoso, para a produção de silagem. Na colheita, as plantas foram separadas em parte aérea e raízes. Da parte aérea do sorgo foram retiradas as panículas, para a avaliação da produção de grãos, e da soja foram retiradas as vagens. Os pesos da matéria seca da parte aérea do sorgo e da soja, dos grãos e dos nódulos, foram obtidos pela secagem dessas partes em estufa a $75^{\circ} \mathrm{C}$, até peso constante. Da matéria seca das folhas obtiveram-se os extratos para a determinação dos nutrientes, após a digestão sulfúrica com $\mathrm{KCl}$ e selênio como catalisador do N (Sarruge \& Haag, 1974) e digestão nitroperclórica dos nutrientes $\mathrm{P}, \mathrm{K}, \mathrm{Ca}, \mathrm{Mg}, \mathrm{Zn}, \mathrm{Cu}$, e Mn (Sarruge \& Haag, 1974), cujas concentrações foram determinadas por espectrometria de emissão de plasma. As concentrações foliares de $\mathrm{N}$ foram determinadas pelo método de Kjeldahl.

Foram amostradas as raízes do sorgo e da soja, para a determinação da colonização micorrízica, depois da clari- 
ficação em KOH e da coloração com azul de tripano, segundo Phillips \& Hayman, (1970); a porcentagem de segmentos de raízes colonizadas foi determinada pelo método da interseção (Giovanetti \& Mosse, 1980).

Os dados foram analisados pelo programa MSTAT-C (Microcomputer Statistical Program, 1989). As médias foram comparadas pelo teste de Duncan. Os dados referentes à porcentagem de colonização das raízes foram transformados por arco seno antes de serem analisados estatisticamente.

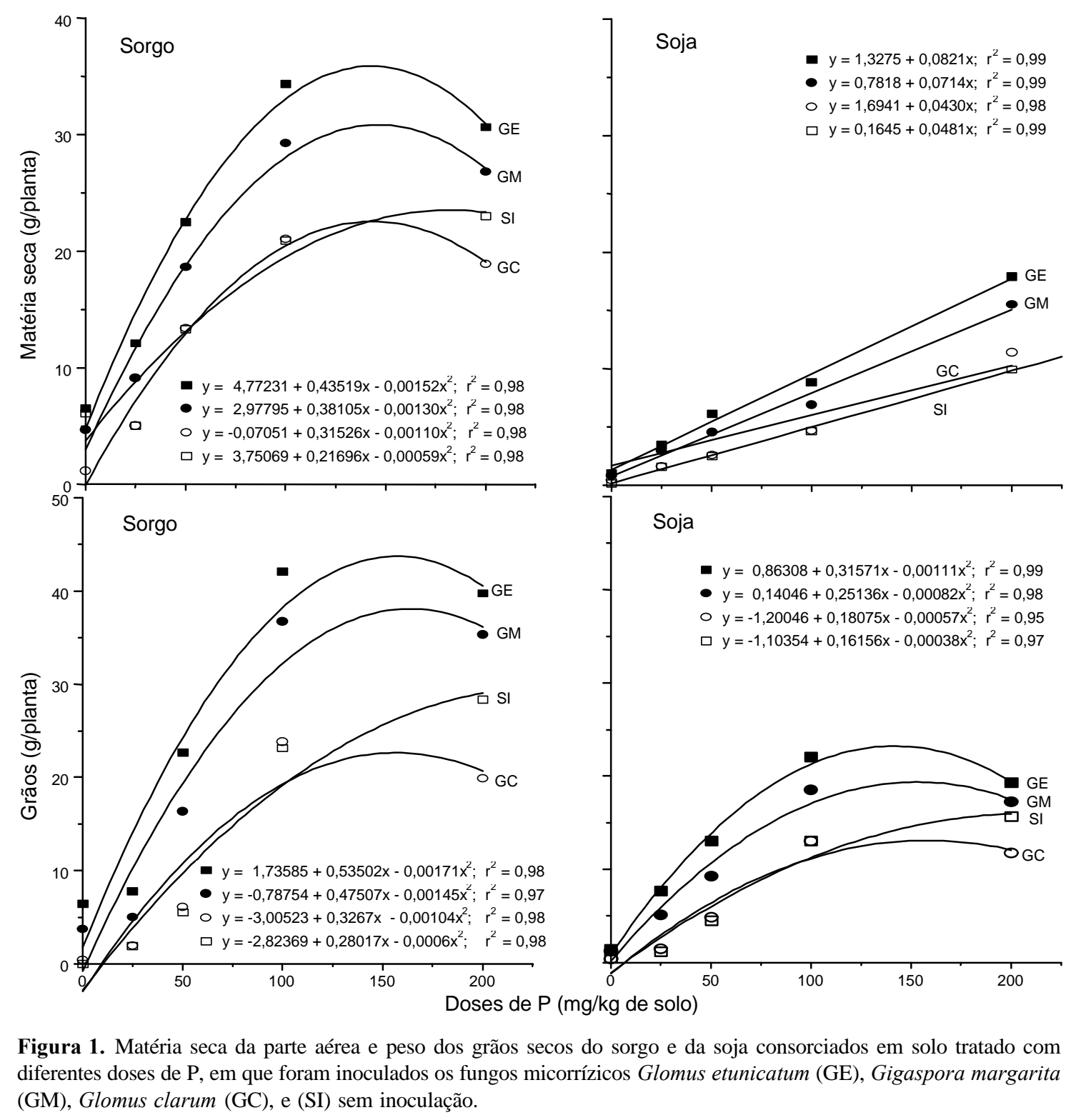

Figura 1. Matéria seca da parte aérea e peso dos grãos secos do sorgo e da soja consorciados em solo tratado com diferentes doses de P, em que foram inoculados os fungos micorrízicos Glomus etunicatum (GE), Gigaspora margarita (GM), Glomus clarum (GC), e (SI) sem inoculação.

\section{Resultados e Discussão}

As respostas de peso da matéria seca da parte aérea (PMSPA) das plantas aos níveis de $\mathrm{P}$ no solo foram quadráticas no sorgo, e lineares e menores na soja, em todos os tratamentos fúngicos (Figura 1). No sorgo, os aumentos máximos foram de 55\%, 33\% e $6 \%$, relativamente aos fungos Glomus etunicatum, Gigaspora margarita e Glomus clarum; estes foram 
alcançados nas doses de 143, 146 e $143 \mathrm{mg} / \mathrm{kg}$ de $\mathrm{P}$, respectivamente. Na soja, o PMSPA aumentou linearmente com as doses de $\mathrm{P}$, o que indica que a dose $200 \mathrm{mg} / \mathrm{kg}$ de P não foi suficiente para maximizar o crescimento desta espécie no consórcio, mesmo nas plantas micorrizadas; isto indica maior exigência de $P$ pela soja em relação ao sorgo.

Tal como foi verificado com o sorgo, Glomus etunicatum e Gigaspora margarita foram as espécies mais eficientes para a soja. Estes efeitos foram observados em todas as doses de P. Quando o PMSPA de cada espécie é expressa em porcentagem da massa total obtida em cada tratamento, verifica-se que a elevação na dose de $\mathrm{P}$ favorece a produção relativa de PMSPA da soja, que em condições subótimas de $\mathrm{P}$ a soja sofre grande competição por esse nutriente. Em condições de baixo P ( 25 e $50 \mathrm{mg} / \mathrm{kg}$ de solo), a massa do sorgo atingiu $85 \%$ e $97 \%$ da matéria seca total das plantas com e sem inoculação, respectivamente. Em alto P (200 mg/kg de solo), o PMSPA da soja atingiu $40 \%$ do total do PMSPA no tratamento com inoculação. Portanto, a aplicação de $\mathrm{P}$ e a inoculação do fungo micorrízico contribui para aumentar a proporção da leguminosa na massa vegetal produzida pelo consórcio sorgo-soja, melhorando, assim, a qualidade desta como forragem ou silagem.

$\mathrm{O}$ efeito do $\mathrm{P}$ sobre a eficiência dos fungos micorrízicos na produção da matéria seca e grãos tem sido verificado por vários autores, em situações diversas (Paula \& Siqueira, 1987; Saggin Júnior et al., 1995). Esses estudos mostraram que doses baixas de $\mathrm{P}$ aumentam a micorrização e a eficiência dos fungos micorrízicos em promover aumento na matéria seca; entretanto, altas doses desse nutriente afetam negativamente a micorrização e a produção de matéria seca. Na soja, apesar de as doses altas de P terem reduzido a colonização micorrízica, não reduziram o PMSPA.

A produção de grãos foi também influenciada por dois outros tratamentos, a saber: adição de $\mathrm{P}$ ao solo, e inoculação com fungos micorrízicos, os quais mostraram interação significativa (Figura 1 ). $O$ peso de grãos secos (PGS) variou em razão da espécie de fungo e das doses de $\mathrm{P}$ adicionadas ao solo; estes fatores são sinergistas. O Glomus etunicatum mostrou maior eficiência em ambas as espécies, em todos os níveis de P. O PGS do sorgo sem inoculação também aumentou com as doses de $\mathrm{P}$, porém mostrou tendência a se estabilizar na dose mais alta de $\mathrm{P}$ (200 mg/kg de solo). Entretanto, no sorgo com inoculação, a aplicação de $\mathrm{P}$ ao solo em doses acima de $150 \mathrm{mg} / \mathrm{kg}$ tenderam a reduzir o PGS. Isto evidencia a importância do P na eficiência de sua utilização pelos fungos micorrízicos nas duas espécies estudadas. Verifica-se que na dose de produção máxima, em torno de $150 \mathrm{mg} / \mathrm{kg}$, a inoculação do Glomus etunicatum aumentou a produção do sorgo em quase $80 \%$ em relação ao controle sem inoculação. Na soja verificaram-se respostas semelhantes e da mesma ordem das obtidas no sorgo.

Os efeitos dos tratamentos nos teores de nutrientes no sorgo e na soja encontram-se na Tabela 1. Os teores de P nas plantas de sorgo e soja aumentaram exponencialmente por causa das doses de $\mathrm{P}$ aplicadas ao solo. As plantas de sorgo e soja que receberam inóculo de Glomus etunicatum mostraram aumento, no teor de P, de 48,2\% e 49,3\%, respectivamente. Este aumento foi verificado nas doses de $\mathrm{P}$ que resultaram em maior concentração desse nutriente nas plantas, de sorgo e soja, a saber: 162 e $172 \mathrm{mg} / \mathrm{kg}$ de $\mathrm{P}$, respectivamente. $\mathrm{O}$ teor de $\mathrm{P}$ nas plantas com inóculo de Gigaspora margarita foi maior no sorgo que na soja, tanto em baixas doses de $\mathrm{P}$ como na dose que resultou no teor máximo de $\mathrm{P}$ nas plantas, indicando assim maior efetividade simbiótica desse fungo micorrízico com o sorgo. No sorgo e na soja verificou-se uma correlação entre o teor de P na parte aérea e o PMSPA $\left(r^{2}=0,89\right.$ e $r^{2}=0,87(p \leq 0,05)$, respectivamente). $\mathrm{O}$ teor de $\mathrm{P}$ na parte aérea também correlacionou-se com a colonização micorrízica das raízes, sendo $r^{2}=0,76$ e r ${ }^{2}=0,72(p \leq 0,05)$, respectivamente, no tocante ao sorgo e à soja.

$\mathrm{O}$ teor de $\mathrm{N}$, na parte aérea, aumentou por causa das doses de P; tal aumento foi mais pronunciado na soja (Tabela 1). Entre os fungos micorrízicos, o Glomus etunicatum foi o mais efetivo, tanto no sorgo como na soja, em promover o aumento do teor de $\mathrm{N}$. A inoculação deste fungo micorrízico aumentou em $31 \%$ e $38 \%$ o teor de $\mathrm{N}$ em relação ao tratamento sem inoculação, nas doses de 123 e $133 \mathrm{mg}$ de $\mathrm{P} / \mathrm{kg}$ de solo, respectivamente, no sorgo e na soja. Em relação ao tratamento sem inoculação, e para atingir o teor máximo de $\mathrm{N}$ na parte aérea, verificou-se que a inoculação de Glomus etunicatum levou a uma 
Tabela 1. Teores de nutrientes nas folhas das plantas de sorgo e soja consorciados, em solo tratado com diferentes doses de $\mathrm{P}$, em que foram inoculados fungos micorrízicos $^{(1)}$.

\begin{tabular}{|c|c|c|c|c|c|c|c|c|c|c|c|c|c|c|c|c|c|}
\hline \multirow{2}{*}{$\begin{array}{c}\text { Doses de } \mathrm{P} \\
(\mathrm{mg} / \mathrm{kg})\end{array}$} & \multirow{2}{*}{ Inoculação } & \multicolumn{2}{|c|}{$\mathrm{N}$} & \multicolumn{2}{|c|}{$\mathrm{P}$} & \multicolumn{2}{|c|}{$\mathrm{K}$} & \multicolumn{2}{|c|}{$\mathrm{Ca}$} & \multicolumn{2}{|c|}{$\mathrm{Mg}$} & \multicolumn{2}{|c|}{$\mathrm{Zn}$} & \multicolumn{2}{|c|}{$\mathrm{Cu}$} & \multicolumn{2}{|r|}{$\mathrm{Mn}$} \\
\hline & & Sorgo & Soja & Sorgo & Soja & Sorgo & Soja & Sorgo & Soja & Sorgo & Soja & Sorgo & Soja & Sorgo & Soja & Sorgo & Soja \\
\hline \multirow{5}{*}{0} & & & & & & $---(g / k$ & & & & & & & & (m & $\mathrm{mg} / \mathrm{kg})-$ & & \\
\hline & Glomus etunicatum & $19,8 \mathrm{~A}$ & $19,0 \mathrm{~A}$ & $0,92 \mathrm{~A}$ & $0,70 \mathrm{~A}$ & $6,8 \mathrm{~A}$ & $4,6 \mathrm{~A}$ & $2,0 \mathrm{~B}$ & $1,5 \mathrm{~B}$ & $1,2 \mathrm{~B}$ & $0,9 \mathrm{~B}$ & $31,24 \mathrm{~A}$ & $28,13 \mathrm{~A}$ & $12,23 \mathrm{~A}$ & $9,65 \mathrm{~A}$ & $32,50 \mathrm{~B}$ & $27,08 \mathrm{AB}$ \\
\hline & Gigaspora margarida & $17,2 \mathrm{~B}$ & $15,8 \mathrm{~B}$ & $0,85 \mathrm{~B}$ & $0,52 \mathrm{~B}$ & $5,2 \mathrm{~B}$ & $3,2 \mathrm{~B}$ & $1,9 \mathrm{~B}$ & $1,0 \mathrm{C}$ & $1,0 \mathrm{C}$ & $0,5 \mathrm{C}$ & $28,14 \mathrm{~A}$ & $22,08 \mathrm{~B}$ & $9,12 \mathrm{~B}$ & $7,12 \mathrm{~B}$ & $26,34 \mathrm{C}$ & $20,35 \mathrm{AB}$ \\
\hline & Glomus clarum & $10,2 \mathrm{C}$ & $12,6 \mathrm{C}$ & $0,38 \mathrm{C}$ & $0,30 \mathrm{C}$ & $3,8 \mathrm{C}$ & $2,0 \mathrm{C}$ & $1,1 \mathrm{~B}$ & $0,6 \mathrm{D}$ & $0,3 \mathrm{D}$ & $0,1 \mathrm{D}$ & $19,12 \mathrm{AB}$ & $34,11 \mathrm{C}$ & $6,58 \mathrm{C}$ & $4,85 \mathrm{C}$ & $14,39 \mathrm{D}$ & $11,11 \mathrm{BC}$ \\
\hline & Sem inoculação & $9,9 \mathrm{C}$ & $11,0 \mathrm{D}$ & $0,30 \mathrm{D}$ & $0,11 \mathrm{D}$ & $2,9 \mathrm{D}$ & $1,2 \mathrm{D}$ & $4,6 \mathrm{~A}$ & $1,8 \mathrm{~A}$ & $1,6 \mathrm{~A}$ & $1,3 \mathrm{~A}$ & $14,20 \mathrm{~B}$ & $10,13 \mathrm{D}$ & $3,21 \mathrm{D}$ & $2,36 \mathrm{D}$ & $39,23 \mathrm{~A}$ & $34,12 \mathrm{~A}$ \\
\hline \multirow[t]{4}{*}{25} & Glomus etunicatum & $22,2 \mathrm{~A}$ & $29,0 \mathrm{~A}$ & $1,24 \mathrm{~A}$ & $0,82 \mathrm{~A}$ & $8,7 \mathrm{~A}$ & $7,4 \mathrm{~A}$ & $4,1 \mathrm{~B}$ & $2,9 \mathrm{~B}$ & $1,4 \mathrm{~B}$ & $1,2 \mathrm{~B}$ & $49,21 \mathrm{~A}$ & $41,12 \mathrm{~A}$ & $26,45 \mathrm{~A}$ & $22,96 \mathrm{~A}$ & $72,13 \mathrm{~B}$ & $50,47 \mathrm{~B}$ \\
\hline & Gigaspora margarida & $19,8 \mathrm{~B}$ & $26,1 \mathrm{~B}$ & $0,95 \mathrm{~B}$ & $0,65 \mathrm{~B}$ & $6,9 \mathrm{~B}$ & $6,0 \mathrm{~B}$ & $3,8 \mathrm{~B}$ & $1,8 \mathrm{C}$ & $1,0 \mathrm{C}$ & $0,9 \mathrm{C}$ & $35,42 \mathrm{~B}$ & $30,34 \mathrm{~B}$ & $19,98 \mathrm{~B}$ & $12,41 \mathrm{~B}$ & $60,42 \mathrm{C}$ & $30,18 \mathrm{C}$ \\
\hline & Glomus clarum & $13,4 \mathrm{C}$ & $20,4 \mathrm{C}$ & $0,51 \mathrm{C}$ & $0,48 \mathrm{C}$ & $4,6 \mathrm{C}$ & $3,6 \mathrm{C}$ & $3,1 \mathrm{~B}$ & $1,0 \mathrm{D}$ & $0,5 \mathrm{D}$ & $0,4 \mathrm{D}$ & $24,16 \mathrm{BC}$ & $=18,15 \mathrm{C}$ & $12,25 \mathrm{C}$ & $8,12 \mathrm{C}$ & $53,71 \mathrm{D}$ & $20,45 \mathrm{D}$ \\
\hline & Sem inoculação & $9,8 \mathrm{D}$ & $19,2 \mathrm{D}$ & $0,42 \mathrm{D}$ & $0,29 \mathrm{D}$ & $3,3 \mathrm{D}$ & $1,9 \mathrm{D}$ & $6,7 \mathrm{~A}$ & $4,2 \mathrm{~A}$ & $1,8 \mathrm{~A}$ & $1,6 \mathrm{~A}$ & $19,21 \mathrm{C}$ & $13,36 \mathrm{D}$ & $8,23 \mathrm{D}$ & $4,25 \mathrm{D}$ & $89,31 \mathrm{~A}$ & $69,13 \mathrm{~A}$ \\
\hline \multirow[t]{4}{*}{50} & Glomus etunicatum & $25,8 \mathrm{~A}$ & $34,4 \mathrm{~A}$ & $1,52 \mathrm{~A}$ & $1,10 \mathrm{~A}$ & $11,2 \mathrm{~A}$ & $9,5 \mathrm{~A}$ & $5,8 \mathrm{~B}$ & $4,5 \mathrm{~B}$ & $1,8 \mathrm{~B}$ & $1,5 \mathrm{~B}$ & $60,23 \mathrm{~A}$ & $52,51 \mathrm{~A}$ & $32,54 \mathrm{~A}$ & $29,75 \mathrm{~A}$ & 101,69B & $68,47 \mathrm{~B}$ \\
\hline & Gigaspora margarida & $21,2 \mathrm{~B}$ & $31,0 \mathrm{~B}$ & $1,23 \mathrm{~B}$ & $0,90 \mathrm{~B}$ & $9,6 \mathrm{~B}$ & $8,0 \mathrm{~B}$ & $5,0 \mathrm{~B}$ & $3,9 \mathrm{C}$ & $1,5 \mathrm{C}$ & $1,1 \mathrm{C}$ & $46,35 \mathrm{~B}$ & $40,18 \mathrm{~B}$ & $24,13 \mathrm{~B}$ & $18,36 \mathrm{~B}$ & $88,40 \mathrm{C}$ & $50,18 \mathrm{C}$ \\
\hline & Glomus clarum & $16,8 \mathrm{C}$ & $24,5 \mathrm{C}$ & $0,86 \mathrm{C}$ & $0,69 \mathrm{C}$ & $5,2 \mathrm{C}$ & $5,1 \mathrm{C}$ & $4,0 \mathrm{~B}$ & $2,7 \mathrm{D}$ & $1,2 \mathrm{D}$ & $0,6 \mathrm{D}$ & $32,12 \mathrm{C}$ & $24,01 \mathrm{C}$ & $16,58 \mathrm{C}$ & $12,29 \mathrm{C}$ & $71,12 \mathrm{D}$ & $41,76 \mathrm{C}$ \\
\hline & Sem inoculação & $15,2 \mathrm{D}$ & $20,8 \mathrm{D}$ & $0,75 \mathrm{D}$ & $0,42 \mathrm{D}$ & $4,1 \mathrm{D}$ & $4,3 \mathrm{D}$ & $8,4 \mathrm{~A}$ & $7,0 \mathrm{~A}$ & $2,4 \mathrm{~A}$ & $2,0 \mathrm{~A}$ & $26,14 \mathrm{C}$ & $19,17 \mathrm{D}$ & $14,23 \mathrm{D}$ & $9,53 \mathrm{D}$ & $115,54 \mathrm{~A}$ & $81,15 \mathrm{~A}$ \\
\hline \multirow[t]{8}{*}{100} & Glomus etunicatun & $29,6 \mathrm{~A}$ & $39,4 \mathrm{~A}$ & $2,01 \mathrm{~A}$ & $1,92 \mathrm{~A}$ & $16,8 \mathrm{~A}$ & $13,0 \mathrm{~A}$ & $6,9 \mathrm{~B}$ & $6,1 \mathrm{~B}$ & $2,6 \mathrm{~B}$ & $1,9 \mathrm{~B}$ & $66,24 \mathrm{~A}$ & $59,85 \mathrm{~A}$ & $36,49 \mathrm{~A}$ & $32,54 \mathrm{~A}$ & $121,35 \mathrm{~B}$ & $90,28 \mathrm{~B}$ \\
\hline & Gigaspora margarida & $23,4 \mathrm{~B}$ & $33,6 \mathrm{~B}$ & $1,86 \mathrm{~B}$ & $1,44 \mathrm{~B}$ & $12,3 \mathrm{~B}$ & $12,0 \mathrm{~B}$ & $6,2 \mathrm{~B}$ & $5,2 \mathrm{C}$ & $2,0 \mathrm{C}$ & $1,5 \mathrm{C}$ & $52,23 \mathrm{~B}$ & $43,12 \mathrm{~B}$ & $27,21 \mathrm{~B}$ & $23,09 \mathrm{~B}$ & $104,23 \mathrm{C}$ & $70,72 \mathrm{C}$ \\
\hline & Glomus clarum & $19,7 \mathrm{C}$ & $28,1 \mathrm{C}$ & $1,03 \mathrm{C}$ & $1,02 \mathrm{C}$ & $7,4 \mathrm{C}$ & $8,9 \mathrm{C}$ & $5,1 \mathrm{~B}$ & $3,6 \mathrm{D}$ & $1,5 \mathrm{D}$ & $0,9 \mathrm{D}$ & $35,12 \mathrm{C}$ & $26,31 \mathrm{C}$ & $18,25 \mathrm{C}$ & $15,63 \mathrm{C}$ & $80,29 \mathrm{D}$ & $51,39 \mathrm{D}$ \\
\hline & Sem inoculação & $18,7 \mathrm{D}$ & $24,0 \mathrm{D}$ & $0,95 \mathrm{C}$ & $0,96 \mathrm{D}$ & $6,5 \mathrm{D}$ & $7,2 \mathrm{D}$ & $9,9 \mathrm{~A}$ & $8,4 \mathrm{~A}$ & $3,4 \mathrm{~A}$ & $2,7 \mathrm{~A}$ & $28,26 \mathrm{C}$ & 24,16D & $16,29 \mathrm{D}$ & $12,36 \mathrm{D}$ & $139,36 \mathrm{~A}$ & $119,41 \mathrm{~A}$ \\
\hline & Glomus etunicatum & $25,4 \mathrm{~A}$ & $37,0 \mathrm{~A}$ & $1,95 \mathrm{~A}$ & $1,80 \mathrm{~A}$ & $15,2 \mathrm{~A}$ & $12,0 \mathrm{~A}$ & $5,6 \mathrm{~B}$ & $5,0 \mathrm{~B}$ & $1,9 \mathrm{~B}$ & $1,6 \mathrm{~B}$ & $57,56 \mathrm{~A}$ & $52,30 \mathrm{~A}$ & $34,85 \mathrm{~A}$ & $29,12 \mathrm{~A}$ & $110,12 \mathrm{~B}$ & $79,48 \mathrm{~B}$ \\
\hline & Gigaspora margarida & $21,2 \mathrm{~B}$ & $32,1 \mathrm{~B}$ & $1,67 \mathrm{~B}$ & $1,22 \mathrm{~B}$ & $11,2 \mathrm{~B}$ & $9,6 \mathrm{~B}$ & $5,1 \mathrm{~B}$ & $3,2 \mathrm{C}$ & $1,1 \mathrm{C}$ & $1,0 \mathrm{C}$ & $54,29 \mathrm{~A}$ & $37,28 \mathrm{~B}$ & $23,54 \mathrm{~B}$ & 19,58B & $92,63 \mathrm{C}$ & $62,93 \mathrm{C}$ \\
\hline & Glomus clarum & $15,4 \mathrm{D}$ & $23,5 \mathrm{D}$ & $0,94 \mathrm{D}$ & $0,94 \mathrm{D}$ & $5,9 \mathrm{D}$ & $7,4 \mathrm{D}$ & $4,0 \mathrm{~B}$ & $2,1 \mathrm{D}$ & $0,7 \mathrm{D}$ & $0,4 \mathrm{D}$ & $28,53 \mathrm{~B}$ & $19,14 \mathrm{D}$ & $17,39 \mathrm{C}$ & $11,46 \mathrm{D}$ & $72,19 \mathrm{D}$ & $44,71 \mathrm{D}$ \\
\hline & Sem inoculação & $19,8 \mathrm{C}$ & $25,1 \mathrm{C}$ & $1,36 \mathrm{C}$ & $1,12 \mathrm{C}$ & $11,8 \mathrm{C}$ & $9,0 \mathrm{C}$ & $11,1 \mathrm{~A}$ & $7,4 \mathrm{~A}$ & $3,9 \mathrm{~A}$ & $2,4 \mathrm{~A}$ & $31,24 \mathrm{~B}$ & $25,31 \mathrm{C}$ & $17,02 \mathrm{C}$ & $15,30 \mathrm{C}$ & $146,25 \mathrm{~A}$ & $123,18 \mathrm{~A}$ \\
\hline
\end{tabular}

${ }^{(1)}$ Médias seguidas de mesma letra nas colunas não diferem significativamente $(\mathrm{P} \leq 0,05)$ pelo teste de Duncan. 
economia, em relação ao $\mathrm{P}$, de 49 e $79 \mathrm{mg}$ de $\mathrm{P} / \mathrm{kg}$ de solo, respectivamente, no sorgo e na soja. O teor de $\mathrm{N}$ na parte aérea mostrou uma correlação com a colonização micorrízica, $\mathrm{r}^{2}=0,80$ e $\mathrm{r}^{2}=0,79(\mathrm{p} \leq 0,05)$, respectivamente, no sorgo e na soja. A aquisição de $\mathrm{N}$ pelas hifas micorrízicas tem sido demonstrada por Azcon \& Barea (1992). Os dados obtidos mostram que tanto no sorgo como na soja a inoculação de fungos micorrízicos aumentou o teor de $\mathrm{N}$ foliar em relação ao tratamento sem inoculação.

A inoculação realizada com fungos micorrízicos também aumentou a concentração de K, Zn e Cu no sorgo e na soja, em relação ao tratamento sem inoculação. Por outro lado, a concentração de $\mathrm{Ca}$, $\mathrm{Mg}$ e Mn foi maior nos tratamentos sem inoculação (Tabela 1), possivelmente devido a um efeito de diluição. Entre as espécies micorrízicas estudadas, o Glomus etunicatum apresentou maior eficiência na absorção dos nutrientes, em todos os níveis de $\mathrm{P}$. Os fungos micorrízicos têm maior importância na absorção de nutrientes de baixa difusão no solo como o P, Cu e Zn (Koide, 1991; Li et al., 1991; Khotari et al., 1991; Marschner \& Dell, 1994). Além disso, os fungos micorrízicos têm acesso a formas solúveis de $\mathrm{P}$ que também estão disponíveis às plantas sem inoculação (Bolan, 1991), como também podem mobilizar $\mathrm{P}$ do solo através da mineralização do $\mathrm{P}$ orgânico (Jaychandran et al., 1992). As concentrações foliares de $\mathrm{Zn}$ e $\mathrm{Cu}$ foram maiores nas plantas de sorgo e soja que receberam o inóculo de fungos micorrízicos, e seus teores aumentaram com os níveis de $\mathrm{P}$, como também foi verificado por Marschner \& Dell (1994).

$\mathrm{O}$ efeito do $\mathrm{P}$ e da inoculação de diferentes fungos na colonização micorrízica de ambas as espécies, encontram-se na Figura 2. A colonização foi

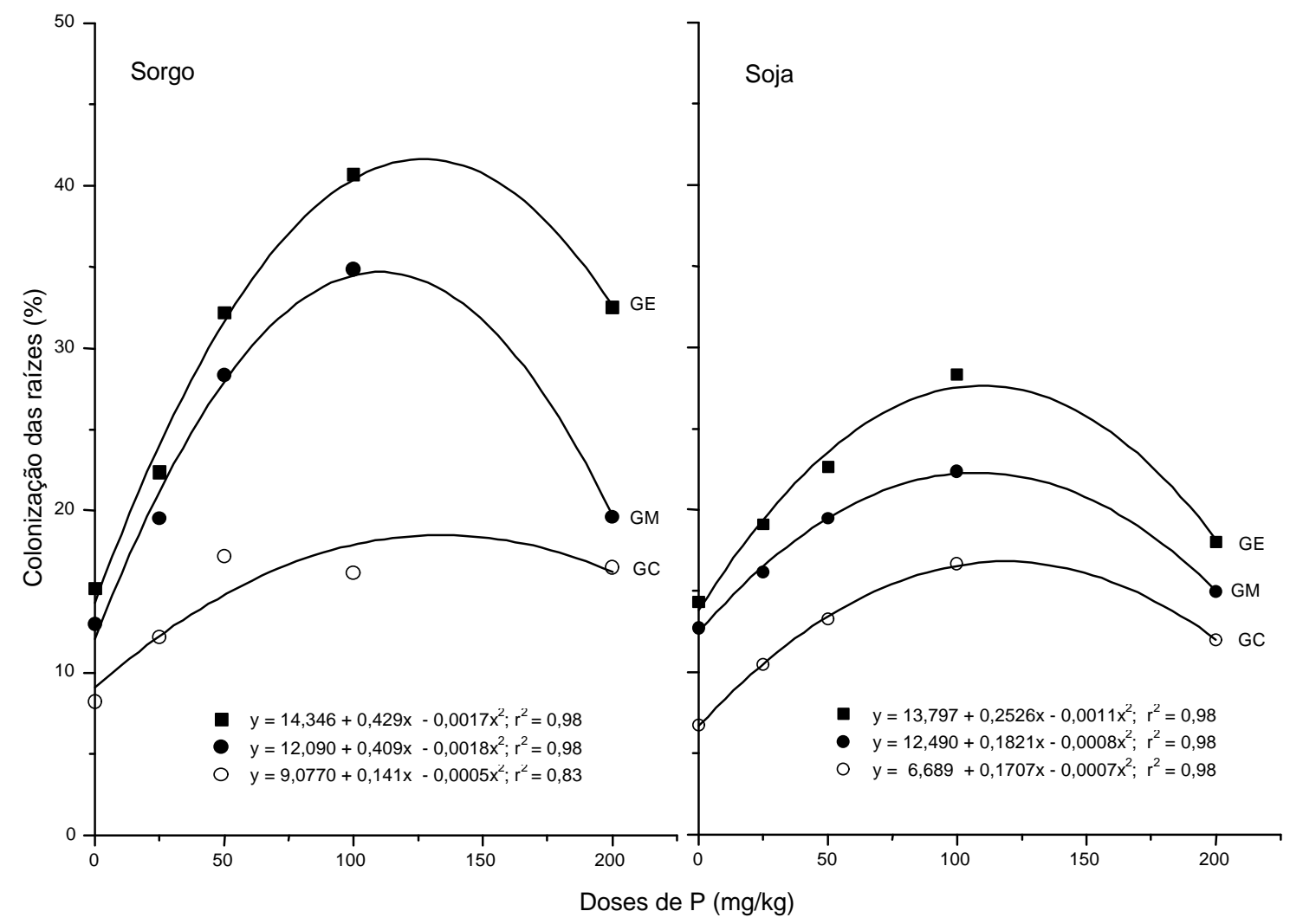

Figura 2. Colonização das raízes do sorgo e da soja consorciados em solo tratado com diferentes doses de $\mathrm{P}$, em que foram inoculados os fungos micorrízicos Glomus etunicatum (GE), Gigaspora margarita (GM) e Glomus clarum (GC). 
máxima com o Glomus etunicatum e Gigaspora margarita, e muito baixa com o Glomus clarum, que se mostrou inefetivo nas espécies estudadas. A colonização micorrízica mostrou valores superiores no sorgo em relação aos da soja, no que respeita aos três fungos micorrízicos. Isto indica maior efetividade dos fungos micorrízicos estudados, referentes ao sorgo. A colonização de diferentes espécies de plantas por Glomus etunicatum, tem mostrado valores entre $36,1 \%$ com relação ao tomateiro (Araújo et al., 1994 ) e 10,8\% com relação ao café (Saggin Júnior et al., 1995). Os valores da colonização micorrízica da soja, verificados em diferentes estudos, têm mostrado ampla faixa de variação. Quanto ao Gigaspora margarita, esses valores têm variado de $62 \%$ a $87 \%$ (Kalil et al., 1994), e quanto ao Glomus clarum, esses valores variaram de 3\% a 59\% (Simpson \& Daft, 1990), o que mostra maior micorrização da Gigaspora margarita, na soja, em relação ao Glomus clarum, como também se verificou pelos dados obtidos neste estudo.

O PMSPA do sorgo mostrou correlação $(\mathrm{p} \leq 0,05)$ positiva com sua colonização micorrízica $\left(\mathrm{r}=0,91^{*}\right)$. Os PGSs do sorgo e da soja também mostraram correlação $(\mathrm{p} \leq 0,05)$ positiva com a colonização micorrízica. Os valores foram $r=0,84$ e $r=0,77$, respectivamente, com relação ao sorgo e à soja. Isto indica que, quanto maior for a colonização micorrízica, maior é o PGS, como também foi verificado por Paula et al. (1990).

$\mathrm{A}$ adição de $\mathrm{P}$ ao solo aumentou o peso dos nódulos secos (PNS), independentemente da inoculação do fungo micorrízico (Figura 3). Doses baixas de P mostraram menores valores do PNS, como também foi observado por Israel (1993). Em relação ao tratamento sem P, a adição de $200 \mathrm{mg}$ de P/kg de solo proporcionou um aumento no PNS em 600, 574, 475 e $441 \mathrm{mg} /$ planta, respectivamente, em relação a Glomus etunicatum, Gigaspora margarita, Glomus clarum e sem inoculação, evidenciando o sinergismo entre o P e a micorriza. Apesar de ter ocorrido uma redução na colonização micorrízica nessa dose de $\mathrm{P}$, a maior disponibilidade de $\mathrm{P}$ no solo pode ter proporcionado um suprimento adequado desse nutriente à soja, aumentando o PNS. O MSPA mostrou uma correlação $(r=0,88)$ significativa $(\mathrm{p} \leq 0,05)$ com PNS. Entre os fungos micorrízicos, o Glomus etunicatum proporcionou maior incremento no PNS em todos os níveis de $\mathrm{P}$, seguido de Gigaspora margarita e Glomus clarum.

Os estudos sobre o efeito da inoculação de fungos micorrízicos no teor de K têm mostrado resultados inconsistentes e de difícil interpretação (Sieverding \& Toro, 1988), e muitas vezes relacionados à absorção de $\mathrm{P}$ (Bethlenfalvay et al., 1989). $\mathrm{A}$ absorção de $\mathrm{Ca}$ pelos fungos micorrízicos (Rhodes \& Gerdeman, 1978) é relativamente baixa, e o seu transporte pode estar associado a grânulos de polifosfatos (White \& Brown, 1979). O baixo teor de Ca nas folhas pode estar associado a esses fatores, como também a um efeito diluição.

Embora os dados obtidos tenham mostrado diminuição na colonização das raízes do sorgo e da soja em altas doses de $\mathrm{P}$, em todas as espécies de fungos micorrízicos estudadas o PMSPA da soja aumentou, mostrando, assim, maior resposta à adição de $\mathrm{P}$, mesmo em níveis que afetam a eficiência da colonização das raízes pelos fungos micorrízicos. Essa ocorrência pode ser decorrente do aumento da nutrição de $\mathrm{P}$, por sua maior disponibilidade em razão da melhor

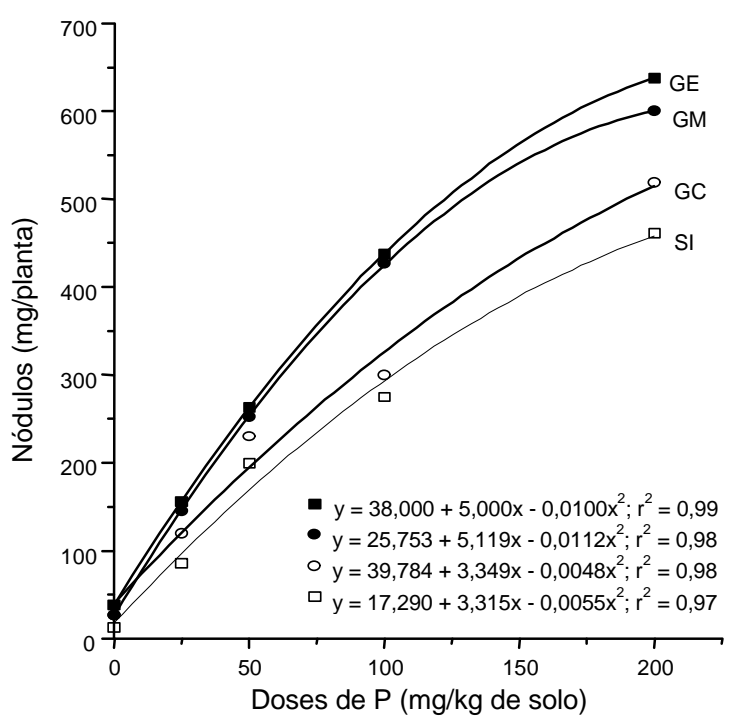

Figura 3. Peso dos nódulos secos das raízes da soja consorciada com sorgo, em solo tratado com diferentes doses de $\mathrm{P}$, em que foram inoculados os fungos micorrízicos Glomus etunicatum (GE), Gigaspora margarita (GM), Glomus clarum (GC), e (SI) sem inoculação. 
nodulação e fixação de N pela soja (Waidyanatha et al., 1979; Lynd et al., 1985). Os estudos sobre a interação fungos micorrízicos-rizóbio indicam que a inoculação desses fungos aumenta a nodulação e a fixação biológica de N. Além disso, a absorção de micronutrientes, como o $\mathrm{Cu}$, também contribui de modo significativo para a fixação do $\mathrm{N}$ (Ross \& Harper, 1970).

\section{Conclusões}

1. O aumento na produção de matéria seca e de grãos do sorgo e da soja consorciados depende das doses de fósforo adicionadas ao solo e da espécie de fungo micorrízico.

2. Glomus etunicatum foi a espécie micorrízica mais eficiente em relação a ambas as espécies vegetais na produção de matéria seca e na produção de grãos.

3. A inoculação, no sorgo e na soja, de fungos micorrízicos, aumenta as concentrações foliares de $\mathrm{N}, \mathrm{P}, \mathrm{K}, \mathrm{Zn}$ e $\mathrm{Cu}$.

\section{Referências}

ARAÚJO, A. P.; SILVA, E. M. R.; ALMEIDA, D. L. Efetividade de fungos endomicorrízicos em tomateiro em diferentes níveis de fósforo no solo. Revista Brasileira de Ciência do Solo, Campinas, v. 18, p. 193-199, 1994.

AZCON, R.; BAREA, J. M. Nodulation, $\mathrm{N}_{2}$ fixation $\left({ }^{15} \mathrm{~N}\right)$ and $\mathrm{N}$ nutrition relationship in mycorhizal or phosphate amended alfalfa plants. Symbiosis, Rehovot, v. 12, n. 1, p. 33-41, 1992.

BETHLENFALVAY, G. J.; FRANSON, R. L.; BROWN, M. S.; MIHARA, K. L. The Glycine - Glomus Bradyrhizobium symbiosis. IX. Nutritional morphological and physiological responses of nodulated soybean of geographic isolates of the mycorhizal fungus Glomus mosseae. Physiologa Plantarum, Copenhagen, v. 76, n. 2 , p. 226-323, 1989.

BOLAN, N. S. A critic review on the role of mycorhizal fungi in the uptake of phosphorus by plants. Plant and Soil, Dordrecht, v. 134, n. 29, p. 189-207, 1991.

GERDEMANN, J. W.; NICHOLSON, T. H. Spores of mycorhizal Endogone species extracted from soil by wet sieving and decanting. British Mycological Society
Transactions, Cambridge, Grã-Bretanha, v. 446, n. 2, p. 235-344, 1963.

GIOVANETTI, M.; MOSSE, B. An evaluation of techniques to measure vesicular arbuscular mycorhizal infection roots. New Phytologist, Cambridge, GrãBretanha, v. 84, n. 3, p. 489-500, 1980.

ISRAEL, W. D. Symbiotic dinitrogen fixation and hostplant growth during development and recovery from phosphorus deficiency. Physiologia Plantarum, Copenhagen, v. 88, n. 2, p. 294-300, 1993.

JAYCHANDRAN, K.; SCHWAB, A. P.; HETRICK, B. A. D. Mineralization of organic phosphorus by vesicular arbuscular mycorhizal fungi. Soil Biology \& Biochemistry, Oxford, v. 2244, n. 9, p. 897-903, 1992.

KALIL, S.; LAYANACHAN, T.E.; TABATABAI, M. A. Mycorhizal dependency and nutrient uptake by improved and unimproved corn and soybean cultivars. Agronomy Journal, Madison, v. 86, n. 6, p. 949-958, 1994.

KHOTARI, S. K.; MARSCHNER, H.; ROMHELD, V. Contribution of VA mycorhizal hyphae in acquisition of phosphorus and zinc by maize in a calcareous soil. Plant and Soil, Dordrecht, v. 131, n. 2, p. 177-185, 1991.

KOIDE, R. T. Nutrient supply, nutrient demand and plant response to mycorhizal infection. New Phytologist, Cambridge, Grã-Bretanha, v. 17, n. 3, p. 365-386, 1991.

LI, X.; GEORGE, E.; MARSCHNER, H. Phosphorus depletion and $\mathrm{pH}$ decrease at the root - soil and hyphae soil interfaces of VA mycorhizal white clover fertilized with ammonium. New Phytologist, Cambridge, Grã-Bretanha, v. 119, n. 3, p. 397-404, 1991.

LYND, J. Q.; RONALD, J. T.; PURCINO, A. A. C. Mycorhiza soil fertility on regrowth, nodulation and nitrogenase activity of siratro (Macroptilum atropurpureum) (DC) Urb.). Journal of Plant Nutrition, New York, v. 8, p. 1047-1059, 1985.

MARSCHNER, H.; DELL, B. Nutrient uptake in mycorhizal symbiosis. Plant and Soil, Dordrecht, v. 159, n. 1, p. 89-102, 1994.

MICROCOMPUTER Statistical Program (MSTAT-C). Lansing : Michigan State University- Institute of International Agriculture, 1989. $280 \mathrm{p}$.

MIRANDA, J. C. C.; HARRIS, P. J.; WILD, A. Effects of soil and plant phosphorus concentrations on vesicular 
arbuscular mycorhiza in sorghum plants. New Phytologist, Cambridge, Grã-Bretanha, v. 112, n. 3, p. 405-410, 1989.

PAULA, M. A.; SIQUEIRA, J. O. Efeito de micorrizas vesicular arbusculares no crescimento, nodulação e acúmulo de N na soja. Pesquisa Agropecuária Brasileira, Brasília, v. 22, n. 2, p. 71-178, fev. 1987.

PAULA, M. A.; SIQUEIRA, J. O.; HOSHIKA, E. Crescimento e produção de soja inoculada com populações de fungos micorrízicos vesículo arbusculares. Revista Brasileira de Ciência do Solo, Campinas, v. 14, n. 2, p. 151-156, 1990.

PHILLIPS, J. M.; HAYMAN, D. S. Improved procedures for cleaning roots and staining parasitic and vesicular arbuscular mycorhizal fungi for rapid assessment for infection. British Mycological Society Transactions, Cambridge, Grã-Bretanha, v. 55, n. 1, p. 158-161, 1970.

RHODES, L. H.; GERDEMAN, J. W. Translocation of calcium and phosphate by external hyphae of vesicular arbuscular mycorrhizae. Soil Science, Baltimore, v. 126, n. 2, p. 125-126, 1978.

ROSS, J. P.; HARPER, J. A. Effect of Endogone mycorhiza on soybean yields. Phytopatology, St. Paul, v. 60, n. 11 , p. $1552-1556,1970$.

SAGGIN JÚNIOR, O. J.; SIQUEIRA, J. O.; GUIMARÃES, P. T. G.; OLIVEIRA, E. Colonização do cafeeiro por diferentes fungos micorrízicos: efeitos na formação das mudas e crescimento em solo fumigado. Revista Brasileira da Ciência do Solo, Campinas, v. 19, n. 2, p. 213-220, 1995.
SARRUGE, J. R.; HAAG, H. P. Análises químicas em plantas. Piracicaba : ESALQ, 1974. 56 p.

SIMPSON, D.; DAFT, M. J. Spore production mycorhizal development in various tropical crop hosts infected with Glomus clarum. Plant and Soil, Dordrecht, v. 121, n. 2, p. 171-178, 1990.

SIEVERDING, E.; TORO, S. Influence of soil water regime on VA mycorrhizae. V. Performance of different VAM fungal species with cassava. Journal of Agronomy and Crop Science, Berlin, v. 161, p. 322-332, 1988.

SMITH, S. E.; GIANINAZZI-PEARSON, V. Physiological interactions between symbionts in vesicular arbuscular mycorhizal plants. Annual Review of Plant Physiology and Plant Molecular Biology, Palo Alto, v. 39, p. 211-244, 1988.

VEJSADOVA, H.; SBLIKOVA, D.; HRSELOVA, H.; VANCURA, V. Effect of the VAM fungus Glomus sp. on the growth and yield of soybean inoculated with Bradyrhizobium japonicum. Plant and Soil, Dordrecht, v. 140, n. 1, p. 121-125, 1992.

WAIDYANATHA, U. P. S.; YOGARATHNAM, N.; ARYARATINE, W. A. Mycorhizal infection on growth and nitrogen fixation of Pueraria and Stylosanthes and uptake of phosphorus from two rock phosphates. New Phytologist, Cambridge, Grã-Bretanha, v. 82, n. 2, p. 147-152, 1979.

WHITE, J. A.; BROWN, M. F. Ultrastructural and X-ray analysis of phosphorus granules in a vesicular arbuscular mycorhizal fungus. Canadian Journal of Botany, Ottawa, v. 57, n. 24, p. 2812-2818, 1979. 